\title{
Low Complexity Clock Synchronization Algorithm for Wireless Sensor Networks with Unknown Delay
}

\author{
Mei Leng and Yik-Chung Wu \\ Department of Electrical and Electronic Engineering \\ The University of Hong Kong \\ Email : \{meileng, ycwu\}@eee.hku.hk
}

\begin{abstract}
In this paper, the problem of clock synchronization is analyzed based on the two-way message exchange mechanism. In order to estimate the clock offset and the clock skew with unknown fixed delay, the maximum likelihood estimator (MLE) and a low-complexity estimator are proposed. Furthermore, their corresponding performance limits and complexities are analyzed. It is found that the MLE achieves the best performance with the price of high complexity, while the newly proposed estimator achieves the same performance as the MLE with low complexity.
\end{abstract}

\section{INTRODUCTION}

Wireless Sensor Network (WSN) was emerged as an important research area in recent years. With its feasibility keep growing rapidly, WSNs have been regarded as fundamental infrastructures for future ubiquitous communications due to a variety of promising potential applications: monitoring the health status of human and environment, control and instrumentation of industrial machines and home appliances, etc. [1] [2]. Most of these applications require collaborative execution of a distributed task amongst a large set of synchronized sensor nodes. Furthermore, data fusion, power management and transmission scheduling require all the nodes running on a common time frame. However, every individual sensor in a WSN has its own clock. Different clocks will drift from each other with time due to many factors, such as imperfection of the oscillators and the environmental changes. This makes clock synchronization between different nodes an indispensable piece of infrastructure.

Clock synchronization is not an easy task in practice due to several unique properties of WSN. The first and most important one is the limited power supply in the low-end sensor nodes. In some cases, sensor nodes are even not rechargeable. To save power, each synchronization procedure should be simple and the frequency of re-synchronization should be low. This fact makes simplicity and accuracy the primary concerns of clock synchronization algorithms for WSNs.

The second challenge of clock synchronization in WSN is the unknown message delays in physical and MAC layers. Kopetz and Ochsenreiter [7] for the first time analyzed the process of message delay and decompose the unknown delay into several components: send time, access time, transmission time, propagation time, reception time and receive time. These delay components can be grouped into two portions: the fixed delay and the random delay. For the fixed delay part, if it is not modeled explicitly, it will be treated as a part of time offset, thus lowering the accuracy of timing parameter estimation.

To deal effectively with the clock synchronization of WSNs, a lot of synchronization protocols have been designed in the past few years. Timing-sync Protocol for Sensor Networks (TPSN) [3] is one of the most widely-used protocols. TPSN is based on the classical two-way message exchange mechanism and corrects the clock offset between two nodes. Unfortunately, the clock skew is not estimated in TPSN, resulting in frequent re-synchronization. Therefore, Noh et al. [9] generalized TPSN to jointly estimate the clock offset and skew, based on an assumption that the fixed delay was known. However, the fixed delay is usually unknown in practice. Although another maximum likelihood-like estimator (GMLLE) that does not require the fixed delay was also derived in [9], the performance of this estimator is not satisfactory.

In this paper, we further generalize TPSN and propose two estimators for joint estimation of the clock offset, clock skew and the fixed delay. One is the maximum likelihood estimator (MLE) whose performance is optimal but complexity is high. The other is a newly proposed estimator whose complexity is low, while can still achieve the same performance as MLE.

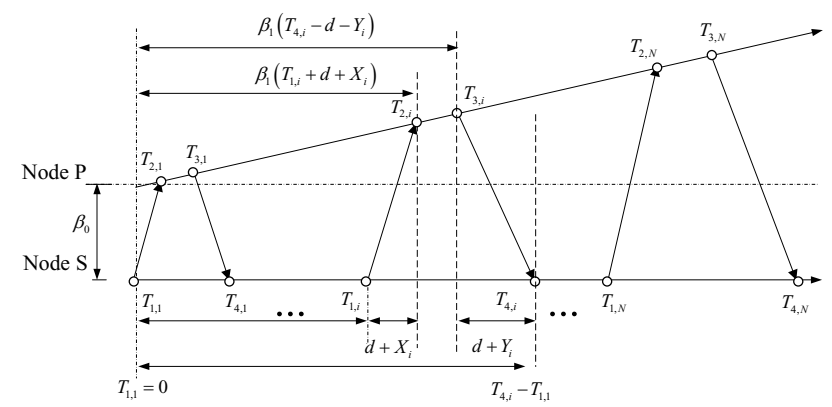

Fig. 1. Two-way time-stamps exchange between two nodes $S$ and $P$.

\section{SYSTEM MODEL}

We consider the synchronization between a parent node $P$ and its child node $S$ based on a two-way timing message exchange mechanism as shown in Fig. 1 . In the $i^{\text {th }}$ round of message exchange, node $S$ sends a request message for clock synchronization to node $P$ at $T_{1, i}$. Node $P$ records its time $T_{2, i}$ at the reception of that message, and replies to node $S$ with a message which contains $T_{2, i}$ and the 
transmission time $T_{3, i}$. Then node $S$ records the reception time of node $P$ 's reply message as $T_{4, i}$. Note that $T_{1, i}$ and $T_{4, i}$ are the time stamps recorded by the clock of node $S$, while $T_{2, i}$ and $T_{3, i}$ are recorded by that of node $P$. After $N$ rounds of message exchange, node $S$ obtains a set of time stamps $\left\{T_{1, i}, T_{2, i}, T_{3, i}, T_{4, i}\right\}_{i=1}^{N}$. The above procedure can be modeled as [9]

$$
\begin{aligned}
& T_{2, i}=\beta_{1} \times T_{1, i}+\beta_{0}+\beta_{1} \times\left(d+X_{i}\right), \\
& T_{3, i}=\beta_{1} \times T_{4, i}+\beta_{0}-\beta_{1} \times\left(d+Y_{i}\right),
\end{aligned}
$$

where $\beta_{0}$ and $\beta_{1}$ represents the clock offset and clock skew, respectively, of node $S$ with respect to node $P$; $d$ stands for the fixed portion of message delay from one node to another, which includes the transmission time, propagation time and reception time [3]. $X_{i}$ and $Y_{i}$ are variable portions of the message delay, comprising the send time, access time and receive time [3]. It is reasonable to assume $X_{i}$ and $Y_{i}$ to be independent and identical distributed (i.i.d.) Gaussian random variables since they are due to numerous independent random processes, and this assumption was experimentally verified in [4]. The goal is to estimate clock offset $\beta_{0}$ and clock skew $\beta_{1}$ based on the observation of a set of time-stamps $\left\{T_{1, i}, T_{2, i}, T_{3, i}, T_{4, i}\right\}_{i=1}^{N}$.

If $d$ is known, it's easy to rewrite the system model (1) and (2) in the standard linear form. Then $\beta_{1}$ and $\beta_{0}$ can be easily estimated [8]. Noh et al. [9] make such assumption and derive the maximum likelihood estimator for joint estimation of clock offset $\beta_{0}$ and clock skew $\beta_{1}$. Unfortunately, in most cases, the value of $d$ will be unknown and even a parameter that needs to be estimated, such as in the context of node localization. Therefore, efficient method for estimating $\beta_{1}$ and $\beta_{0}$ with unknown delay $d$ is of great interest.

In the following, we first present the MLE for joint estimation of clock skew $\beta_{1}$, clock offset $\beta_{0}$ and fixed delay $d$. The MLE represents the optimal solution and provides a performance benchmark. However, it is computationally expensive because of the calculation of the fixed delay, and may not be applicable for the low-end terminals in WSNs. Thus, a low-complexity estimator is then proposed in Section $\mathrm{IV}$, and this estimator is shown having the same performance as MLE while its complexity is very low.

\section{Maximum Likelihood Estimator}

\section{A. MLE}

Based on the system model (1) and (2), we will derive the MLE for the joint estimation of clock offset $\beta_{0}$, clock skew $\beta_{1}$ and fixed delay $d$. Rewrite (1) and (2) as

$$
\begin{aligned}
& \frac{1}{\beta_{1}} \cdot T_{2, i}=T_{1, i}+\frac{\beta_{0}}{\beta_{1}}+d+X_{i} \\
& \frac{1}{\beta_{1}} \cdot T_{3, i}=T_{4, i}+\frac{\beta_{0}}{\beta_{1}}-d-Y_{i} .
\end{aligned}
$$

Defining $\theta_{1}=1 / \beta_{1}$ and $\theta_{0}=\beta_{0} / \beta_{1}$, and stacking all the time-stamps in matrix form, the model becomes

$$
\underbrace{\left[\begin{array}{c}
T_{1,1} \\
\vdots \\
T_{1, N} \\
-T_{4,1} \\
\vdots \\
-T_{4, N}
\end{array}\right]}_{\triangleq \mathbf{T}_{a}}+d \cdot \mathbf{1}=\underbrace{\left[\begin{array}{cc}
T_{2,1} & -1 \\
\vdots & \vdots \\
T_{2, N} & -1 \\
-T_{3,1} & 1 \\
\vdots & \vdots \\
-T_{3, N} & 1
\end{array}\right]}_{\triangleq \mathbf{T}_{p}} \underbrace{\left[\begin{array}{c}
\theta_{1} \\
\theta_{0}
\end{array}\right]}_{\triangleq \boldsymbol{\Theta}}-\underbrace{\left[\begin{array}{c}
X_{1} \\
\vdots \\
X_{N} \\
Y_{1} \\
\vdots \\
Y_{N}
\end{array}\right]}_{\triangleq \mathbf{Z}},
$$

where $\mathbf{1}=[1, \cdots, 1]^{T}$ with dimension $2 N \times 1$.

Since i.i.d. random delays $X_{i}$ and $Y_{i}$ follow Gaussian distribution (i.e., $X_{i} \sim \mathcal{N}\left(0, \sigma^{2}\right), Y_{i} \sim \mathcal{N}\left(0, \sigma^{2}\right)$ ), the probability density function (PDF) can be written as

$$
\ln f\left(\mathbf{T}_{a}, \mathbf{T}_{p} ; \boldsymbol{\Theta}, d\right)=\ln \frac{N}{2 \pi \sigma^{2}}-\frac{\left\|\mathbf{T}_{a}+d \mathbf{1}-\mathbf{T}_{p} \boldsymbol{\Theta}\right\|^{2}}{2 \sigma^{2}} .
$$

For a fixed $d$, the MLE of $\Theta$ is [8]

$$
\hat{\boldsymbol{\Theta}}(d)=\left(\mathbf{T}_{p}^{H} \mathbf{T}_{p}\right)^{-1} \mathbf{T}_{p}^{H}\left(\mathbf{T}_{a}+d \mathbf{1}\right) .
$$

Plugging (7) into (6), and ignoring some irrelevant constants, we arrive at the compressed likelihood function with only one parameter $d$. Denote $\mathbf{P}=\mathbf{I}_{2 N}-\mathbf{T}_{p}\left(\mathbf{T}_{p}^{H} \mathbf{T}_{p}\right)^{-1} \mathbf{T}_{p}^{H}$, and note that $\mathbf{P}^{H} \mathbf{P}=\mathbf{P}$, the likelihood function is given by

$$
\Lambda(d)=\left\|\mathbf{P}\left(\mathbf{T}_{a}+d \mathbf{1}\right)\right\|^{2} .
$$

Taking derivative over the likelihood function (8) with respect to $d$, and setting the result to be zero, it can be shown that the estimator for $d$ is

$$
\hat{d}=-\frac{1}{2} \cdot \frac{\mathbf{1}^{H} \mathbf{P} \mathbf{T}_{a}+\mathbf{T}_{a}^{H} \mathbf{P} \mathbf{1}}{\mathbf{1}^{H} \mathbf{P} \mathbf{1}} .
$$

Putting the estimated $\hat{d}$ back into (7), we finally get the estimator of $\beta_{1}$ and $\beta_{0}$ as $\hat{\beta}_{1}=1 /[\hat{\boldsymbol{\Theta}}(\hat{d})]_{1}$ and $\hat{\beta}_{0}=$ $[\hat{\boldsymbol{\Theta}}(\hat{d})]_{2} /[\hat{\boldsymbol{\Theta}}(\hat{d})]_{1}$, where $[\mathbf{v}]_{i}$ denotes $i^{\text {th }}$ element of vector $\mathbf{v}$.

\section{B. Cramer-Rao Lower Bound}

Noh et al. [9] presented the CRLB for joint estimation of clock skew $\beta_{1}$ and clock offset $\beta_{0}$ under the assumption that the fixed delay $d$ was known. When the fixed delay is included as a parameter, we need to re-derive the CRLB for each parameter in the joint estimation.

The PDF in (6) can be rewritten as

$$
\begin{gathered}
\ln f\left(\left\{T_{1, i}, T_{2, i}, T_{3, i}, T_{4, i}\right\}_{i=1}^{N} ; \beta_{1}, \beta_{0}, d\right)=\ln \frac{N}{2 \pi \sigma^{2}}-\frac{1}{2 \sigma^{2}} . \\
\sum_{i=1}^{N}\left[\left(\frac{T_{2, i}}{\beta_{1}}-T_{1, i}-\frac{\beta_{0}}{\beta_{1}}-d\right)^{2}+\left(T_{4, i}-\frac{T_{3, i}}{\beta_{1}}+\frac{\beta_{0}}{\beta_{1}}-d\right)^{2}\right] .
\end{gathered}
$$

Then the Fisher Information Matrix is given by

$$
\begin{aligned}
\operatorname{FIM}\left(\beta_{1}, \beta_{0}, d\right) & =\left[\begin{array}{ccr}
-\mathbf{E} \frac{\partial^{2} \ln f}{\partial \beta_{1}^{2}} & -\mathbf{E} \frac{\partial^{2} \ln f}{\partial \beta_{1} \partial \beta_{0}} & -\mathbf{E} \frac{\partial^{2} \ln f}{\partial \beta_{1} \partial d} \\
-\mathbf{E} \frac{\partial^{2} \ln f}{\partial \beta_{0}^{2}} & -\mathbf{E} \frac{\partial^{2} \ln f}{\partial \beta_{0} \partial \beta_{1}} & -\mathbf{E} \frac{\partial^{2} \ln f}{\partial \beta_{0} \partial d} \\
-\mathbf{E} \frac{\partial^{2} \ln f}{\partial d^{2}} & -\mathbf{E} \frac{\partial^{2} \ln f}{\partial d \partial \beta_{1}} & -\mathbf{E} \frac{\partial^{2} \ln f}{\partial d \partial \beta_{0}}
\end{array}\right] \\
& =\frac{1}{\sigma^{2}}\left[\begin{array}{ccc}
A & B & C \\
B & \frac{2 N}{\beta_{1}^{2}} & 0 \\
C & 0 & 2 N
\end{array}\right],
\end{aligned}
$$


where $A \triangleq \frac{1}{\beta_{1}^{4}} \sum_{i=1}^{N}\left[\beta_{1}^{2}\left(T_{1, i}+d\right)^{2}+\beta_{1}^{2} \sigma^{2}+\left(T_{3, i}-\beta_{0}\right)^{2}\right]$, $B \triangleq \frac{1}{\beta_{1}^{3}} \sum_{i=1}^{N}\left[\beta_{1}\left(T_{1, i}+d\right)+\left(T_{3, i}-\beta_{0}\right)\right]$ and $C \triangleq$ $\frac{1}{\beta^{2}} \sum_{i=1}^{N}\left[\beta_{1}\left(T_{1, i}+d\right)-\left(T_{3, i}-\beta_{0}\right)\right]$. By inverting the matrix (10), it can be shown that the CRLB for each parameter is

$$
\begin{aligned}
& \operatorname{CRLB}\left(\beta_{1}\right)=\frac{2 N \sigma^{2}}{2 N A-\beta_{1}^{2} B^{2}-C^{2}}, \\
& \operatorname{CRLB}\left(\beta_{0}\right)=\frac{\sigma^{2} \beta_{1}^{2}\left(2 N A-C^{2}\right)}{2 N\left(2 N A-\beta_{1}^{2} B^{2}-C^{2}\right)}, \\
& \operatorname{CRLB}(d)=\frac{\sigma^{2}\left(2 N A-\beta_{1}^{2} B^{2}\right)}{2 N\left(2 N A-\beta_{1}^{2} B^{2}-C^{2}\right)} .
\end{aligned}
$$

Remark 1: In the two-way message exchange mechanism, the synchronizing node $S$ initiates the exchange process and decides how many rounds are needed. In each round, node $S$ decides the time $T_{1, i}$ to send the request message and node $P$ decides the time $T_{3, i}$ to reply when it receives the request message. Thus in each round, the values of time stamp $T_{1, i}$ and $T_{3, i}$ are controlled by the two nodes separately and should be seen as the system input, while $T_{2, i}$ and $T_{4, i}$ as the corresponding outputs. Once the inputs and statistics of the random delay are fixed, the CRLB should be fixed and independent of the system outputs. Therefore, the CRLB derived above depends only on the time stamps $\left\{T_{1, i}, T_{3, i}\right\}_{i=1}^{N}$ and the random delay variance. However, the CRLB derived in [9] was based on all the time stamps $\left\{T_{1, i}, T_{2, i}, T_{3, i}, T_{4, i}\right\}_{i=1}^{N}$, and that CRLB may change according to different realizations of $\left\{T_{2, i}, T_{4, i}\right\}_{i=1}^{N}$.

\section{Proposed Low COMPleXity Estimator}

\section{A. Proposed estimator}

In the previous section, the MLE for joint estimation of the clock offset, clock skew and the fixed delay is derived. The performance of the MLE is good but its complexity is high. In the following, a low complexity estimator that has the same performance as the MLE is derived.

Rewriting the original model by adding (1) to (2), the modified model is given by

$$
T_{2, i}+T_{3, i}=\beta_{1} \times\left(T_{1, i}+T_{4, i}\right)+2 \beta_{0}+\beta_{1} \times\left(X_{i}-Y_{i}\right) .
$$

Dividing the above equation by $\beta_{1}$, and stacking all the timestamps in matrix form, the model becomes

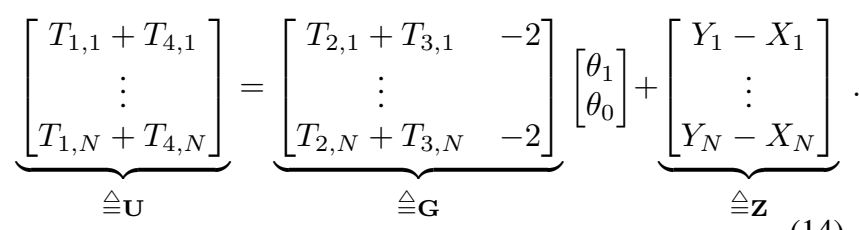

Since the i.i.d. random delay $X_{i}$ and $Y_{i}$ follow Gaussian distribution with variance $\sigma^{2}, Z_{i} \triangleq\left(Y_{i}-X_{i}\right) \sim \mathcal{N}\left(0,2 \sigma^{2}\right)$. The MLE for $\Theta$ is

$$
\hat{\boldsymbol{\Theta}}=\left(\mathbf{G}^{H} \mathbf{G}\right)^{-1} \mathbf{G}^{H} \mathbf{U} .
$$

The estimator for $\beta_{1}$ and $\beta_{0}$ are then given by $\hat{\beta}_{1}=1 /[\hat{\boldsymbol{\Theta}}]_{1}$ and $\hat{\beta}_{0}=[\hat{\boldsymbol{\Theta}}]_{2} /[\hat{\boldsymbol{\Theta}}]_{1}$.
In order to estimate $d$, the estimated clock skew and clock offset are plugged into the original model (1) and (2),

$$
\begin{aligned}
& T_{2, i}-\hat{\beta}_{1} \times T_{1, i}-\hat{\beta}_{0}=\hat{\beta}_{1}\left(d+X_{i}\right), \\
& T_{3, i}-\hat{\beta}_{1} \times T_{4, i}-\hat{\beta}_{0}=-\hat{\beta}_{1}\left(d+Y_{i}\right) .
\end{aligned}
$$

Subtract (17) from (16), and after some simple manipulations, we have

$$
\frac{1}{\hat{\beta}_{1}}\left[\left(T_{2, i}-T_{3, i}\right)-\hat{\beta}_{1}\left(T_{1, i}-T_{4, i}\right)\right]=2 d+\left(X_{i}+Y_{i}\right) .
$$

Since $X_{i}$ and $Y_{i}$ are i.i.d. Gaussian random variables with variance $\sigma^{2},\left(X_{i}+Y_{i}\right) \sim \mathcal{N}\left(0,2 \sigma^{2}\right)$. The MLE for delay $d$ can be easily shown to be [8]

$$
\hat{d}=\frac{1}{2 N}\left[\frac{1}{\hat{\beta}_{1}} \sum_{i=1}^{N}\left(T_{2, i}-T_{3, i}\right)-\sum_{i=1}^{N}\left(T_{1, i}-T_{4, i}\right)\right] .
$$

Remark 2: Although the fixed delay is a useful parameter in some cases, it is a nuisance parameter if only the clock synchronization is considered. By using the proposed estimator, the calculation of $d$ can be skipped if only $\beta_{1}$ and $\beta_{0}$ are of interest.

\section{B. Performance bound for the proposed estimator}

Strictly speaking, for a set of data generated from a specific system model, there should be a unique and lowest performance bound for any estimator dealing with these data. The CRLB given in Section III-B is such kind of lowest bound. However, if the data is manipulated in the process of estimation such that the system model for estimation is changed, we need to know whether the manipulation causes deterioration in the performance limit. Therefore, a performance bound for the proposed estimator is needed.

From the model $\mathbf{U}=\mathbf{G} \boldsymbol{\Theta}+\mathbf{Z}$ in (14), the PDF of $\mathbf{U}$ is $\ln f(\mathbf{U} ; \boldsymbol{\Theta})=-\frac{N}{2} \ln \left(2 \pi \sigma^{2}\right)-\frac{1}{2 \sigma^{2}}\|\mathbf{U}-\mathbf{G} \boldsymbol{\Theta}\|^{2}$. Then the Fisher information matrix can be derived as [8]

$$
\operatorname{FIM}\left(\beta_{1}, \beta_{0}\right)=\frac{1}{2 \sigma^{2}}\left[\begin{array}{cc}
D & 2 B \\
2 B & \frac{4 N}{\beta_{1}^{2}}
\end{array}\right]
$$

where $D=\frac{1}{\beta_{1}^{2}} \sum_{i=1}^{N}\left\{\left[\left(T_{1, i}+d\right)+\frac{1}{\beta_{1}}\left(T_{3, i}-\beta_{0}\right)\right]^{2}+3 \sigma^{2}\right\}$. Inverting this $2 \times 2$ matrix, the performance bounds for the clock skew $\beta_{1}$ and the clock offset $\beta_{0}$ can be found as

$$
\begin{aligned}
\operatorname{PB}_{p}\left(\beta_{1}\right) & =\frac{2 \sigma^{2} N}{N D-\beta_{1}^{2} B^{2}}, \\
\operatorname{PB}_{p}\left(\beta_{0}\right) & =\frac{\sigma^{2} \beta_{1}^{2} D}{2 N D-2 \beta_{1}^{2} B^{2}} .
\end{aligned}
$$

\section{PERFORMANCE BOUNDS COMPARISON}

To compare the performance bound $\operatorname{PB}_{p}\left(\beta_{1}\right)$ of the modified system with $\operatorname{CRLB}\left(\beta_{1}\right)$, the difference of the denominators of (11) with (19) is first computed. After some manipulation, it can be shown that

$2 N A-C^{2}-N D=\frac{N^{2}}{\beta_{1}^{2}}\left[\frac{1}{N} \sum_{i=1}^{N} V_{i}^{2}-\left(\frac{1}{N} \sum_{i=1}^{N} V_{i}\right)^{2}-\sigma^{2}\right]$ 
TABLE I

COMPLEXITY COMPARISON

\begin{tabular}{|c|c||c|c|c|}
\hline Estimator & parameter & number of $+/-$ & number of $\times$ & number of $\div$ \\
\hline \multirow{3}{*}{ MLE } & $\beta_{0}(d)$ & $20 N-4$ & $16 N+7$ & 0 \\
\cline { 2 - 5 } & $\beta_{1}(d)$ & $20 N-4$ & $16 N+6$ & 1 \\
\cline { 2 - 5 } & $d$ & $8 N^{3}+19 N^{2}+14 N-6$ & $8 N^{3}+11 N^{2}+15 N+6$ & 3 \\
\hline \multirow{3}{*}{ Proposed Estimator } & $\beta_{0}$ & $7 N-5$ & $5 N+3$ & 1 \\
\cline { 2 - 5 } & $\beta_{1}$ & $7 N-3$ & $2 N+4$ & 1 \\
\cline { 2 - 5 } & $d\left(\beta_{1}\right)$ & $4 N-1$ & 2 & \\
\hline
\end{tabular}

where $V_{i}=\left(T_{1, i}+d\right)-\frac{1}{\beta_{1}}\left(T_{3, i}-\beta_{0}\right)$. Without loss of generality, suppose $T_{1, i}=i H$ and $T_{3, i}=i K$, where $H$ and $K$ are the gap between neighboring time stamps in $T_{1, i}$ and $T_{3, i}$, respectively. Then, the normalized difference between (11) and (19) can be calculated as

$$
\begin{aligned}
\frac{\operatorname{PB}_{p}\left(\beta_{1}\right)-\operatorname{CRLB}\left(\beta_{1}\right)}{\operatorname{CRLB}\left(\beta_{1}\right)} & =\frac{\left(H-\frac{K}{\beta_{1}}\right)^{2}-\frac{12 \sigma^{2}}{N^{2}-1}}{\left(H+\frac{K}{\beta_{1}}\right)^{2}+\frac{36 \sigma^{2}}{N^{2}-1}} \\
& <\frac{\left(H-\frac{K}{\beta_{1}}\right)^{2}}{\left(H+\frac{K}{\beta_{1}}\right)^{2}} .
\end{aligned}
$$

Since $H$ and $K$ are usually of similar values to keep the two-way message exchange in order, and the clock skew is generally close to 1 , the normalized difference will be a very small constant. For example, suppose $H=25, K=30$ and $N=6$ (the other parameters are the same as in the simulation section), the normalize difference is approximately 0.0078. Thus, the proposed estimator for clock skew $\beta_{1}$ has almost the same performance limit as MLE. Simulation results shown in Section VII further verify that the performance bound $\mathrm{PB}_{p}\left(\beta_{1}\right)$ of the proposed estimator is indistinguishable from the $\operatorname{CRLB}\left(\beta_{1}\right)$.

Similarly, the normalized difference between (12) and (20) is calculated as (shown in the Appendix)

$$
\begin{aligned}
& \frac{\operatorname{PB}_{p}\left(\beta_{0}\right)-\operatorname{CRLB}\left(\beta_{0}\right)}{\operatorname{CRLB}\left(\beta_{0}\right)} \\
& <\frac{15(N+1)^{2}}{\left(N^{2}-1\right)+\left(4 N^{2}+6 N+2\right) \cdot \frac{\left(H+\frac{K}{\beta_{1}}\right)^{2}}{\left(H-\frac{K}{\beta_{1}}\right)^{2}}} \\
& \approx \frac{15}{1+4 \cdot \frac{\left(H+\frac{K}{\beta_{1}}\right)^{2}}{\left(H-\frac{K}{\beta_{1}}\right)^{2}}} .
\end{aligned}
$$

With the same reason stated above, the normalized difference between $\operatorname{PB}_{p}\left(\beta_{0}\right)$ and $\operatorname{CRLB}\left(\beta_{0}\right)$ will be a very small constant. Using the same parameter setting as above, the normalized difference is approximately 0.0064 . Thus we can conclude that the proposed estimator for clock offset $\beta_{0}$ has almost the same performance limit as MLE, and this is further verified by the simulation results.

\section{COMPLEXITY COMPARISON}

To compare the complexity of the two estimators, their number of operations are shown in Table I. The symbol $L(Q)$ has been used to indicate that the calculation of parameter $L$ depends on the pre-calculated parameter $Q$. As shown in Table I, for MLE, estimation of $\beta_{0}$ and $\beta_{1}$ depends on the estimated $d$, while the calculation of $\hat{d}$ takes large number of operations. Thus the MLE for estimation of $\beta_{0}$ and $\beta_{1}$ is computational expensive. On the other hand, the proposed estimator has much lower complexity.

\section{Simulation RESUlts}

As mentioned in the introduction, Noh et al. proposed an estimator named GMLLE in [9], and it can be written as $\hat{\beta}_{1}=\left[\left(T_{2, N}-T_{2,1}\right)^{2}+\left(T_{3, N}-T_{3,1}\right)^{2}\right] /\left[\left(T_{1, N}-\right.\right.$ $\left.\left.T_{1,1}\right)\left(T_{2, N}-T_{2,1}\right)+\left(T_{3, N}-T_{3,1}\right)\left(T_{4, N}-T_{4,1}\right)\right]$ and $\hat{\beta}_{0}=$ $\frac{1}{2 N} \sum_{i=1}^{N}\left[\left(T_{2, i}+T_{3, i}\right)-\hat{\beta}_{1}\left(T_{1, i}+T_{4, i}\right)\right]$. Here, simulations are carried out to compare the performances of the three estimators (MLE, the proposed estimator and GMLLE). The parameters used in the simulation are $d \in(0,10], \beta_{1} \in$ $[0.9,1.1]$ and $\beta_{0} \in[-10,10]$. In each simulation run, $d$, $\beta_{1}$ and $\beta_{0}$ are uniformly drawn from their respective range. 10000 simulation runs were performed to obtain the average performance of each point in the figures.

Fig. 2 shows the mean squared error (MSE) for estimation of the clock offset $\beta_{0}$. As shown in the figure, the proposed estimator achieve the best performance as MLE and its performance bound overlaps with CRLB, while the performance of GMLLE degraded with respect to the CRLB. Fig. 3 shows MSE for estimation of the clock skew $\beta_{1}$. The same conclusions as in Fig. 2 can be drawn.

\section{CONCLUSIONS}

Clock synchronization for WSN in the presence of Gaussian delay was discussed based on the two-way message exchange mechanism. The MLE for joint estimation of clock skew, clock offset and fixed delay was first derived. The MLE achieves good performance but its complexity is high. Then a novel and low-complexity estimator was proposed that achieves the same performance as the MLE, while its complexity is very low. The proposed estimator was also compared with the GMLLE in [9]. It was found that the proposed estimator performs much better than GMLLE. Therefore, among the three estimators (MLE, the proposed estimator, and GMLLE), the proposed estimator represents the most efficient clock synchronization algorithm for WSNs. 


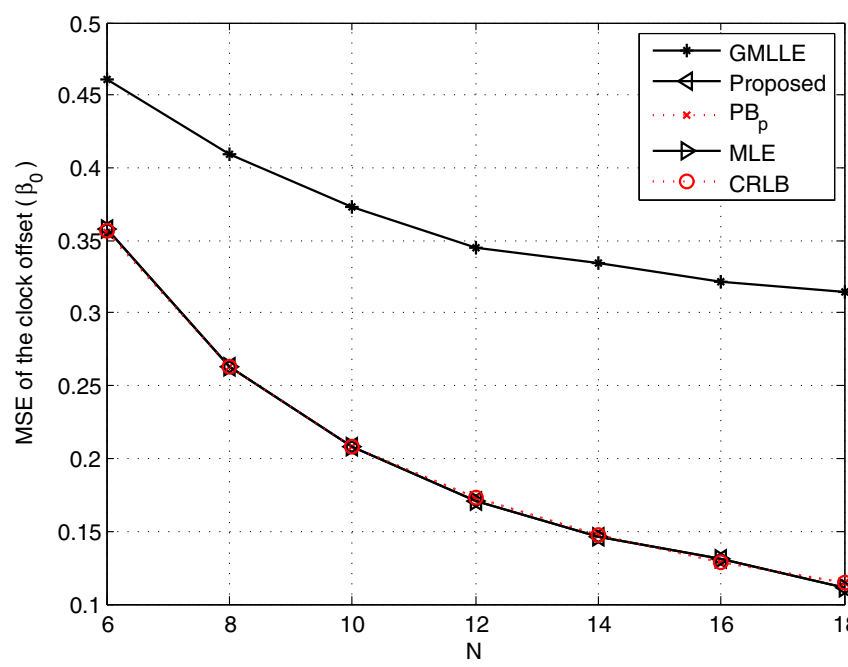

Fig. 2. MSE of estimated clock offset $\hat{\beta_{0}}$ with respect to the number of rounds of message exchange $N$.

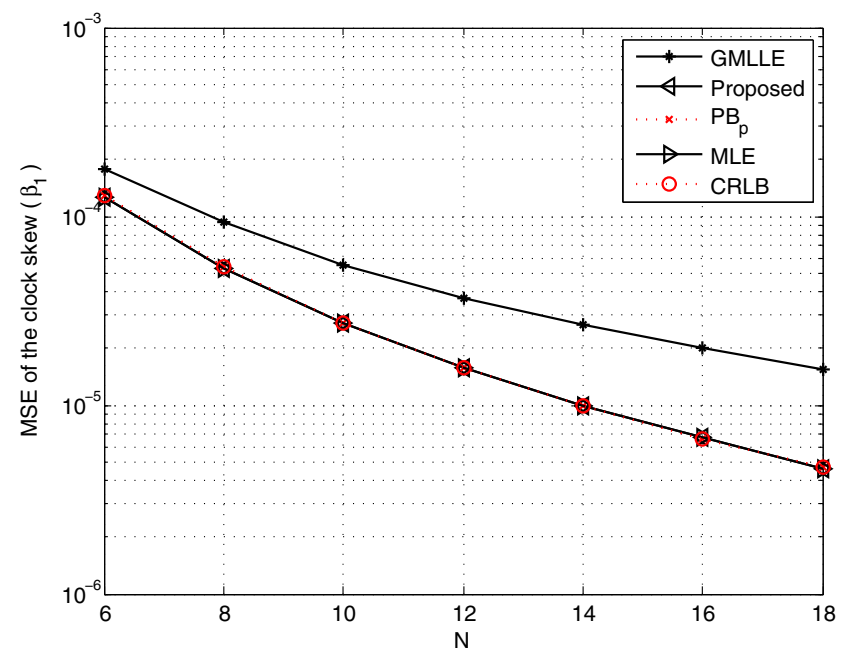

Fig. 3. MSE of estimated clock skew $\hat{\beta_{1}}$ with respect to the number of rounds of message exchange $N$.

\section{APPENDIX \\ PROOF OF $(22)$}

From (12) and (20), it can be shown that

$$
\begin{aligned}
& \frac{\operatorname{PB}_{p}\left(\beta_{0}\right)-\operatorname{CRLB}\left(\beta_{0}\right)}{\operatorname{CRLB}\left(\beta_{0}\right)} \\
& =\frac{\left[\left(H-\frac{K}{\beta_{1}}\right)^{2}\left(N^{2}-1\right)-12 \sigma^{2}\right] \cdot \beta_{1}^{2} B^{2}}{\left[\left(H+\frac{K}{\beta_{1}}\right)^{2}\left(N^{2}-1\right)+36 \sigma^{2}\right]\left\{N D+\frac{N^{2}}{12 \beta_{1}^{2}}\left[\left(H-\frac{K}{\beta_{1}}\right)^{2}\left(N^{2}-1\right)-\sigma^{2}\right]\right\}} .
\end{aligned}
$$

Since $N D=\frac{N^{2}}{\beta_{1}^{2}}\left[\frac{1}{6}\left(2 N^{2}+3 N+1\right)\left(H+\frac{K}{\beta_{1}}\right)^{2}+3 \sigma^{2}+\triangle\right]$ and $\beta_{1}^{2} B^{2}=\frac{N^{2}}{\beta_{1}^{2}}\left[\frac{1}{4}(N+1)^{2}\left(H+\frac{K}{\beta_{1}}\right)^{2}+\triangle\right]$, where $\triangle=(N+1)\left(H+\frac{K}{\beta_{1}}\right)+\left(d-\frac{\beta_{0}}{\beta_{1}}\right)^{2}$ is a positive term smaller than $(N+1)^{2}\left(H+\frac{K}{\beta_{1}}\right)^{2}$, we have

$$
\begin{aligned}
& N D>\frac{N^{2}}{\beta_{1}^{2}}\left[\frac{1}{6}\left(2 N^{2}+3 N+1\right)\left(H+\frac{K}{\beta_{1}}\right)^{2}+3 \sigma^{2}\right], \\
& \beta_{1}^{2} B^{2}<\frac{N^{2}}{\beta_{1}^{2}} \cdot \frac{5}{4}(N+1)^{2}\left(H+\frac{K}{\beta_{1}}\right)^{2} .
\end{aligned}
$$

Therefore, the normalized difference between $\operatorname{PB}_{p}\left(\beta_{0}\right)$ and $\operatorname{CRLB}\left(\beta_{0}\right)$ can be shown to satisfy that following inequality

$$
\begin{aligned}
& \frac{\operatorname{PB}_{p}\left(\beta_{0}\right)-\operatorname{CRLB}\left(\beta_{0}\right)}{\operatorname{CRLB}\left(\beta_{0}\right)} \\
& <\frac{15(N+1)^{2}\left(H-\frac{K}{\beta_{1}}\right)^{2}}{\left(N^{2}-1\right)\left(H-\frac{K}{\beta_{1}}\right)^{2}+\left(4 N^{2}+6 N+2\right)\left(H+\frac{K}{\beta_{1}}\right)^{2}} .
\end{aligned}
$$

\section{REFERENCES}

[1] I. F. Akyildiz, W. Su, Y. Sankarasubramaniam, E. Cayirci, "Wireless sensor networks: a survey", Cumputer Networks, vol. 38, no. 4, pp. 393-422, Mar. 2002.

[2] N. Bulusu and S. Jha, Wireless Sensor Networks: A Systems Perspective, Artech House, 2005.

[3] S. Ganeriwal, R. Kumar and M. B. Srivastava, "Timing-sync protocol for sensor networks", in Proc. of 1st International Conference on Embeded Networks Sensor Systems. New York: ACM Press, 2003, pp. 138-149.

[4] J. Elson, L. Girod, D. Estrin, "Fine-Grained Network Time Synchronization using Reference Broad- casts", in Proc. of the Fifth Symposium on Operating System Design and Implementation Symp, Boston, MA, Dec. 2002. pp. 147-163.

[5] M. Maroti, B. Kusy, G. Simon, A. Ledeczi, "The Flooding Time Synchronization Protocol". In Proc. of the 2nd International Conference on Embedded Networked Sensor Systems. New York: ACM Press. 2004, pp. 39-49.

[6] H. S. Abedel-Ghaffar, "Analysis of synchronization algorithms with time-out control over networks with experimentally symmetric delays". IEEE Trans. On Communications. vol. 50, no. 10, pp. 1652-1661. Oct. 2002.

[7] H. Kopetz and W. Ochsenreiter, "Clock synchronization in distributed real-time systems". in IEEE Trans. on Computers., vol. 36, no. 8, pp. 933-940, Aug. 1987.

[8] S. Kay, Fundamentals of Statistical Signal Processing: Estimation Theory, Englewood Cliffs, NJ: Prentice-Hall, 1993.

[9] K. L. Noh, Q. M. Chaudhari, E. Serpedin and B. W. Suter, "Novel clock phase offset and skew estimation using two-way timing message exchanges for wireless sensor networks," IEEE Trans. on Communications. vol. 55, no. 4, pp. 766-777, April 2007. 